\title{
Administración de proyectos: el ordenamiento territorial de la vivienda asistida
}

Project management: land use planning for assisted living

Administração de projetos:planejamento do uso da terra para vida assistida

Luis Alberto Salgado Cabrera

Tecnológico Nacional de México / Instituto Tecnológico de Acapulco, México penanbury@gmail.com https://orcid.org/0000-0001-8769-2262

Jacqueline Bringas Ramírez

Tecnológico Nacional de México / Instituto Tecnológico de Acapulco, México bringasjacqueline@gmail.com https://orcid.org/0000-0002-4041-5679

Levid Ezer Nava Solis*

Tecnológico Nacional de México / Instituto Tecnológico de Acapulco, México nava_solis@yahoo.com.mx https://orcid.org/0000-0001-9786-1728

Lucero Way Mendez*

Tecnológico Nacional de México / Instituto Tecnológico de Acapulco, México Iwaym10@gmail.com https://orcid.org/0000-0002-1674-419X

Autores de correspondencia* 


\title{
Resumen
}

El programa de vivienda asistida del Gobierno federal plantea una alianza con el beneficiario, quien contribuye con sus conocimientos empíricos en el levantamiento de su futura habitación, y la Comisión Nacional de Vivienda. El resultado es obtener tres tipos de capitales: uno para la compra de material de calidad de construcción, otro para la mano de obra y, por último, el capital de apoyo de un asistente técnico ya sea para la construcción, reforzamiento, remodelación o ampliación del hábitat, y con ello lograr una disminución en el rezago habitacional. Aquí se presenta la viabilidad de dicha estrategia en el poblado de Acamixtla, Taxco, Guerrero.

Palabras clave: administración de proyectos, autoconstrucción, vivienda asistida.

\begin{abstract}
The federal government's assisted housing program establishes an alliance with the beneficiary, who contributes with their empirical knowledge in the lifting of their future room, and the National Housing Commission. The result is to obtain three types of capital: one for the purchase of quality construction material, another for labor and, finally, the support capital of a technical assistant either for construction, reinforcement, remodeling or expansion. habitat, and thereby achieve a decrease in the housing gap. The feasibility of this strategy in the town of Acamixtla, Taxco, Guerrero is presented here.
\end{abstract}

Keywords: project management, self-construction, assisted living.

\section{Resumo}

O programa de habitação assistida do governo federal estabelece aliança com o benefici ário, que contribui com seu conhecimento empírico na elevação de seu futuro quarto, e a Comissão Nacional de Habitação. O resultado é a obtenção de três tipos de capital: um para aquisição de material de construção de qualidade, outro para mão-de-obra e, por ú ltimo, o capital de apoio de um assistente técnico quer para construção, reforço, remodelação ou expansão. Habitat, e assim conseguir uma diminuição no fosso habitacional. A viabilidade desta estratégia na cidade de Acamixtla, Taxco, Guerrero é apresentada aqui.

Palavras-chave: gerenciamento de projetos, autoconstrução e vida assistida.

Fecha Aceptación: Octubre 2021 


\section{Introducción}

Los asentamientos humanos tienen su génesis en las costumbres alimenticias, culturales y de construcción de vivienda. Cumplir con estas tres dimensiones es cumplir con lo que debe tener un espacio digno y cómodo para habitar.

En junio de 2006, México da a conocer en el Diario Oficial de la Federación por primera vez la Ley de Vivienda, la cual introduce una conceptualización binaria de productor y de constructor, es decir, que con sus propias manos autoproduce el material e insumos que utilizará para después construir por su cuenta lo que será su vivienda. Con el pasar del tiempo dicha conceptualización se va modificando de manera notoria según los criterios de pensamiento, creencias e ideas, sobre todo con los habitantes que presentan pobreza extrema por la falta de más de dos recursos básicos y también de los residentes que viven en periferias o en lugares muy apartados, tal y como es el caso del poblado de Acamixtla. Las pocas familias que ahí viven no cuentan con los recursos que debe tener una casa habitación en su interior. La Organización de las Naciones Unidas [ONU] (2016), como resultado de las peticiones de las partes involucradas, de los formadores de opinión, activistas, especialistas, líderes comunitarios, maestros, entre otros, presenta la una Nueva Agenda Urbana para los próximos 20 años. El Programa de Naciones Unidas para los Asentamientos Humanos (ONU-Habitat) es una agencia de la ONU que incluye y visibiliza los objetivos acordados durante la conferencia denominada Habitat III.

Durante varias décadas o generaciones, estas familias se vieron sometidas a un escaso o nulo ingreso monetario, y esto provocó el aislamiento social. Aunque el estilo de vida de estas familias no es observado de forma negativa internamente, ya que lo consideran como natural; han aceptado vivir con carencias elementales, es decir, la herencia de una cultura social que sus ancestros les han regalado. Taxco está identificado arquitectónicamente como patrimonio de la humanidad por sus características únicas y especiales que son el resultado de fenómenos naturales. Así pues, se trata de un atractivo natural y cultural.

La pobreza, de acuerdo con la definición que otorga la ONU (s. f.):

Va más allá de la falta de ingresos y recursos para garantizar unos medios de vida sostenibles. Es un problema de derechos humanos. Entre las distintas manifestaciones de la pobreza figuran el hambre, la malnutrición, la falta de una vivienda digna y el acceso limitado a otros servicios básicos como la educación o la salud (párr. 2). 


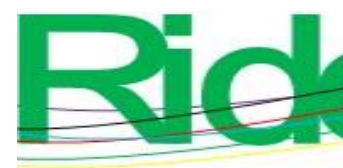

Revista Iberoamericana para la Investigación y el Desarrollo Educativo ISSN 2007 - 7467

Se requieren diferentes recursos para realizar las tareas del proyecto y lograr el objetivo planteado. Los recursos incluyen personas, materiales, equipos, instalaciones, etc. Los recursos humanos son las personas con conocimientos técnicos o habilidades específicas. También se requieren ciertas cantidades de cada tipo de recurso con conocimientos específicos en determinados periodos a lo largo del proyecto (Gido y Clements, 2012, p. 7). Se estudió Acamixtla por considerarse una localidad de pobreza extrema, de acuerdo con investigaciones hechas por el Consejo Nacional de Evaluación de la Política a de Desarrollo Social [Coneval] (2015), cuyo criterio es que una persona vive pobreza extrema cuando tiene tres o más carencias sociales, de seis posibles, y, además, su ingreso total es menor que la línea de bienestar mínimo. La población en esta situación dispone de un ingreso tan bajo que, aun si lo dedicase por completo a la adquisición de alimentos, no podría acceder a aquellos que componen la canasta alimentaria. Guerrero en 2015 se encontraba dentro de los 15 municipios con mayor registro de pobreza extrema, en el lugar número cuatro, y con un resultado de $87.7 \%$ (Coneval, 2015).

Las viviendas en esta comunidad siempre han sido levantadas por los propios habitantes, es decir, no tienen ningún elemento de los mencionados en el conjunto anterior. Según Krajewski, Malhotra, y Ritzman (2008), “administración de proyectos es un método sistematizado y progresivo para definir, organizar, planificar, monitorear y controlar" (p. 71). La administración en este proyecto de vivienda pretende que se reduzcan los costos en la construcción y, como consecuencia, que la vivienda sea accesible a personas con menores ingresos.

\section{Marco teórico}

Es difícil comprender la arraigada vida de la periferia de Acamixtla si no se toma en cuenta un dato importante sobre los habitantes de la comunidad: están acostumbrados a vivir distintas carencias sociales y no consideran aspectos que puedan transformar sus viviendas y así lograr habitar un lugar digno. La ONU (2017) prevé que la población urbana mundial se duplicará para el año 2050, lo que hará de la urbanización una de las tendencias más transformadoras en el siglo XXI. 
Las poblaciones, las actividades económicas, las interacciones sociales y culturales, así como las repercusiones ambientales y humanitarias, se concentran cada vez más en las ciudades, y ello plantea enormes problemas de sostenibilidad en materia de vivienda, infraestructura, servicios básicos, seguridad alimentaria, salud, educación, empleos decentes, seguridad y recursos naturales, entre otros (ONU, 2017, p. 3).

Si bien aún resta mucho tiempo para que se cumpla esta fecha, sin embargo, es de prioridad dar una solución inmediata a las condiciones que actualmente tienen las viviendas. En la ya citada Nueva Agenda Urbana (ONU, 2017) se afirma que la persistencia de múltiples formas de pobreza, las desigualdades crecientes y la degradación ambiental siguen siendo uno de los principales obstáculos para el desarrollo sostenible en todo el mundo: la exclusión social y económica y la segregación espacial son una realidad irrefutable en las ciudades y los asentamientos humanos (p. 3).

En Acamixtla este proyecto pretende cambiar las viviendas en la periferia: ofrecer un espacio limpio, amplio, cómodo, pero sobre todo digno de habitar para poder dar cumplimiento a las variables de transformación social.

La Constitución Política de los Estados Unidos Mexicanos (28 de mayo de 2021), en su artículo cuatro, establece que "toda familia tiene derecho a disfrutar de vivienda digna y decorosa. La Ley establecerá los instrumentos y apoyos necesarios a fin de alcanzar tal objetivo". Este maridaje entre la administración de proyectos y el ordenamiento territorial de vivienda asistida atiende la proyección espacial con eficiencia dentro del entorno sociocultural de los habitantes de Acamixtla.

Guerrero, según datos arrojados por el Instituto Nacional de Estadística y Geografía [Inegi] (2016), es la entidad 12 y está conformada de 81 municipios.

Figura 1. Ubicación de Guerrero y sus municipios.

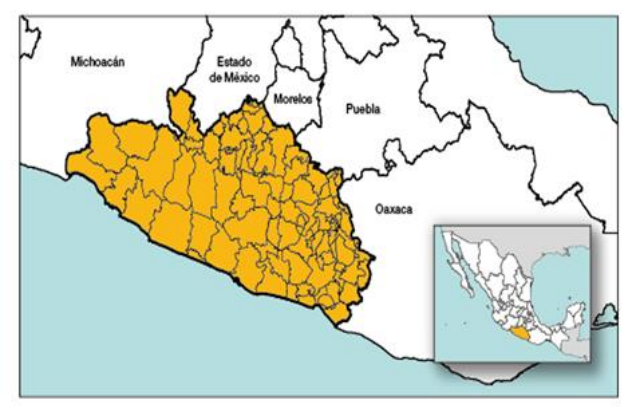

Fuente: Inegi (2016) 

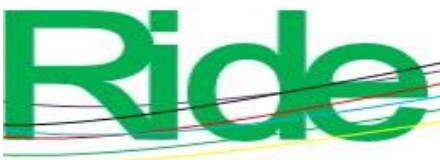

Revista Iberoamericana para la Investigación y el Desarrollo Educativo ISSN 2007 - 7467

Taxco está identificado con la clave municipal número 55. Se encuentra al norte del estado de Guerrero. Tiene una altitud de 1800 metros sobre el nivel del mar.

Figura 2. Ubicación del municipio de Taxco, Guerrero, México

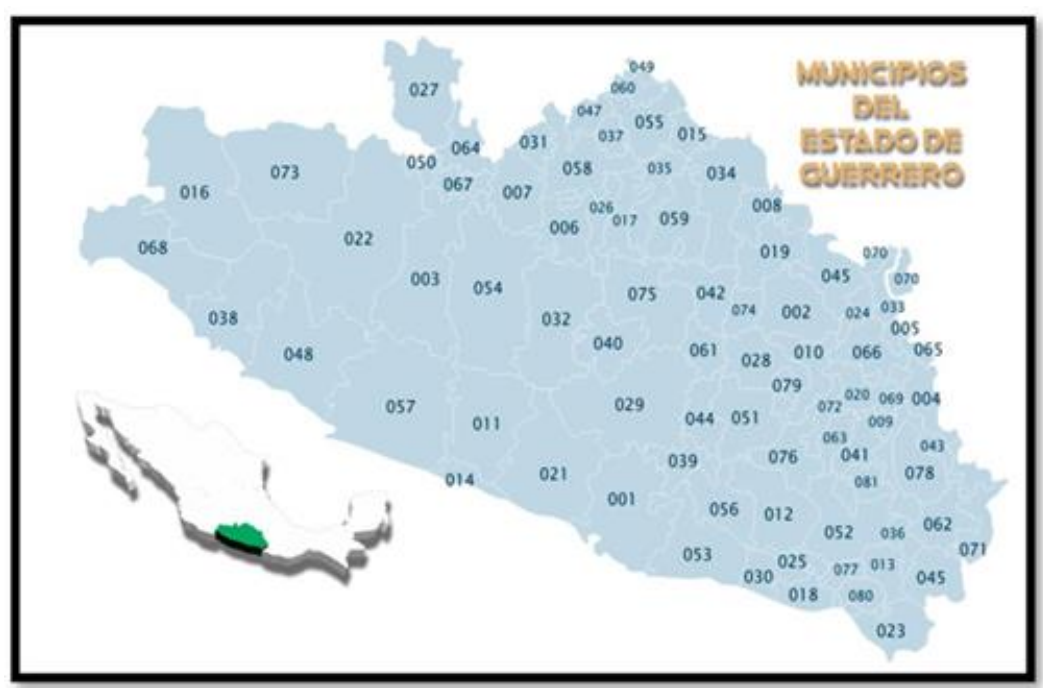

Fuente: México Real (2017)

Acamixtla es una localidad que corresponde al municipio de Taxco, cuenta con una extensión territorial de 347 kilómetros cuadrados y representa $0.54 \%$ del total de la superficie de Guerrero.

Figura 3. Ubicación de la localidad de Acamixtla, Taxco, Guerrero

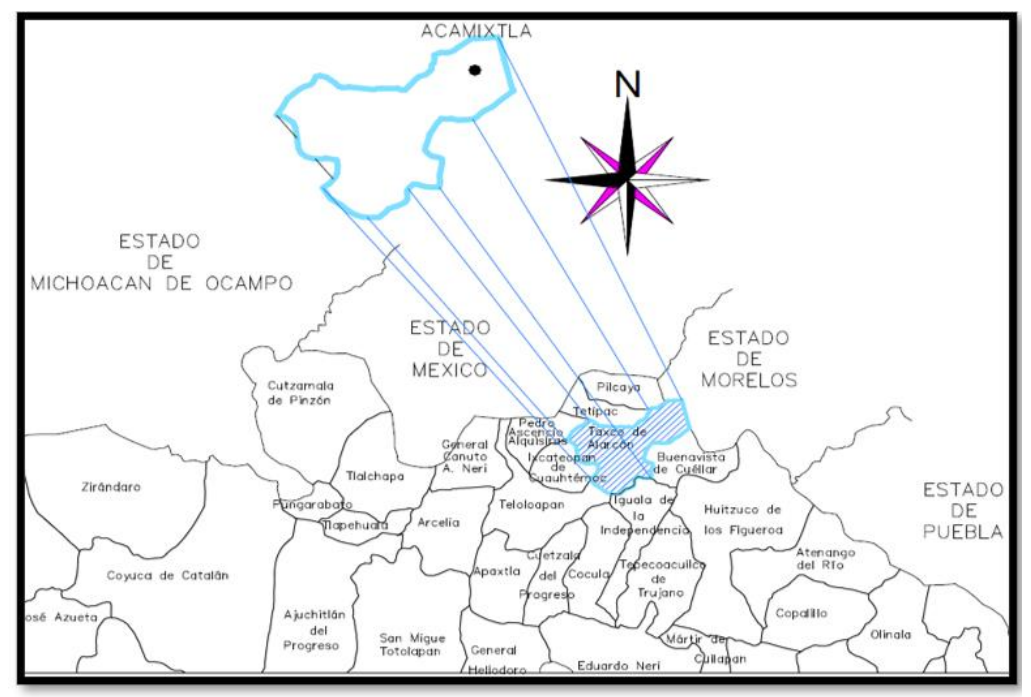

Fuente: VH Construcciones y Asesoría en Ingeniería (2009, p. 16)

La Secretaría de Desarrollo Agrario, Territorial y Urbano [Sedatu] (26 de junio de 2020), en conjunto con la Comisión Nacional de Vivienda, para arribar progresivamente a la vivienda adecuada, lanzó tres estrategias diferentes, una de las cuales impulsaba la 
autoproducción asistida con el propósito de combatir el rezago habitacional existente en las comunidades más pobres de este país.

La administración de proyectos de vivienda debe comprender las actividades que se van a ejecutar y cada uno de sus recursos que son requeridos: provisiones, materiales, tiempo, humanos y factores económicos.

La administración eficaz y eficiente de un proyecto consiste en la realización de un conjunto de actividades (interrelacionadas, en serie o en paralelo) involucradas en un proceso de consecución de recursos (físicos, humanos y financieros, principalmente). Estas actividades son llevadas a cabo por miembros de un equipo interdisciplinario, dirigido por un gerente de proyecto, para alcanzar objetivos relacionados con la programación, los costos, el desempeño técnico y los entregables del proyecto (Garrido y Carrillo, 2013, p. 75)

\section{Vivienda asistida}

Dentro del documento del Programa Nacional de Vivienda 2019-2024, la Sedatu (2019) señala que, en la actualidad, millones de mexicanos viven en asentamientos precarios que carecen de servicios y equipamientos mínimos, donde los tiempos y costos de transporte para tener acceso al trabajo, la educación y el abasto son elevados. Por eso se implementará un programa de construcción de vivienda popular que garantice este derecho y genere miles de empleos. El derecho a la ciudad y a la vivienda involucra aspectos fundamentales del hábitat como la seguridad jurídica, la disponibilidad de infraestructura, servicios y equipamientos urbanos, de salud, educación y trabajo (Sedatu, 2019, p.12). Aun cuando se sigan presentando paulatinamente algunas mejoras respecto a las necesidades de vivienda, sigue habiendo vulnerabilidad por la situación económica tan precaria dentro de los espacios marginales. Para Bedolla, Miranda, Bedolla y Sánchez (2021): "La población, en algunos casos, no cuenta con una cultura de percepción, por lo que es aún más vulnerable a los efectos producidos por los fenómenos naturales" (p. 11). Las poblaciones siguen creciendo desproporcionadamente y dicho crecimiento es muy difícil de frenar. En consecuencia, el espacio para habitar, al tener que compartirse con más personas, es menor, lo que impide el desarrollo de un ambiente sano y de alegría. En el estado de Guerrero existe un alto grado de incidencia de marginación que ha llevado a la pérdida de identidad cultural. Entre los lugares más icónicos de este problema se encuentra el municipio de Taxco y su localidad de Acamixtla, porque sigue vigente su magia arquitectónica, sin embargo, la urbanización 
y la creciente población ha hecho que los asentamientos irregulares se multipliquen de manera acelerada. Así, la probabilidad de perder la belleza que caracteriza este espacio es cada vez más alta.

\section{Autoproducción de vivienda asistida}

"Existe un consenso en definir la productividad, en términos generales, como la relación entre productos e insumos, haciendo de este indicador una medida de la eficacia con el cual la organización utiliza sus recursos para producir bienes finales" (Medianero, 2016, p, 24). La autoproducción asistida es un tipo de vivienda que pretende buscar una solución a la carente existencia de oportunidades que tienen las familias como las del poblado de Acamixtla para poder construir un lugar habitable y adecuado, capaz de integrarse al medio, a las costumbres y a las dinámicas de la comunidad; lograr adaptar y habitar el territorio sin perder la identidad y tradición de sus pobladores.

El ingreso es la principal variable que determina el grado de pobreza en una sociedad y es la variable que ha permitido dar seguimiento a las estadísticas de pobreza por 25 años en México (Foster, Greer y Thorbecke, 1984, citados en Aguilar, Caamal y Portillo, 2018). El poblado de Acamixtla se distingue por su valiosa participación social y cultural. Allí, las personas han demostrado tener la habilidad de generar por cuenta propia la producción básica en alimentos, ropa e insumos para su vivienda. Es decir, se trata de una población que consume constantemente los recursos que autoproduce, sobre todo las personas que no tienen los recursos económicos suficientes.

Según el Project Management Institute [PMI] (2017), planificar el involucramiento poblacional es el proceso de desarrollar enfoques para involucrar a los interesados del proyecto, con base en sus necesidades, expectativas, intereses y el posible impacto de este. El beneficio clave es que proporciona un plan factible para interactuar de manera eficaz con los interesados (p. 594). Por su continuidad dentro del propio esquema, la vinculación en cada etapa está en relación con la anterior, porque se repite el ciclo cada vez que el individuo necesita ser otra vez un autoproductor. Todo proyecto en su trayectoria de vida presenta hitos más sobresalientes, tal como se muestra en la figura 4. 
Figura 4. Los grupos de proceso en la administración de proyectos

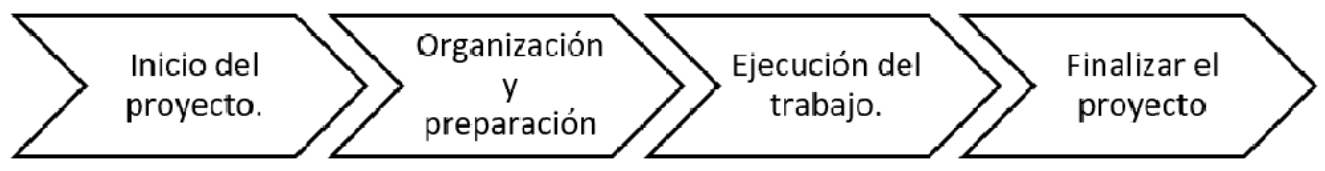

Fuente: Elaboración propia con base en PMI (2017)

Rodríguez (2001, citado en Shek, 2013), por su parte, identifica el método sistémico como una herramienta indispensable para los gerentes de proyectos, ya que este permite analizar todos los componentes y determinar la relación e importancia de ellos.

Cuando se finaliza el proyecto, el entregable es un espacio cómodo y digno para satisfacer esa emergencia del poblado en extrema pobreza de Acamixtla. Por lo anterior, el mando ejecutivo brinda la ayuda para construir, remodelar o mejorar su hábitat.

Figura 5. Estructura genérica del ciclo de vida del proyecto

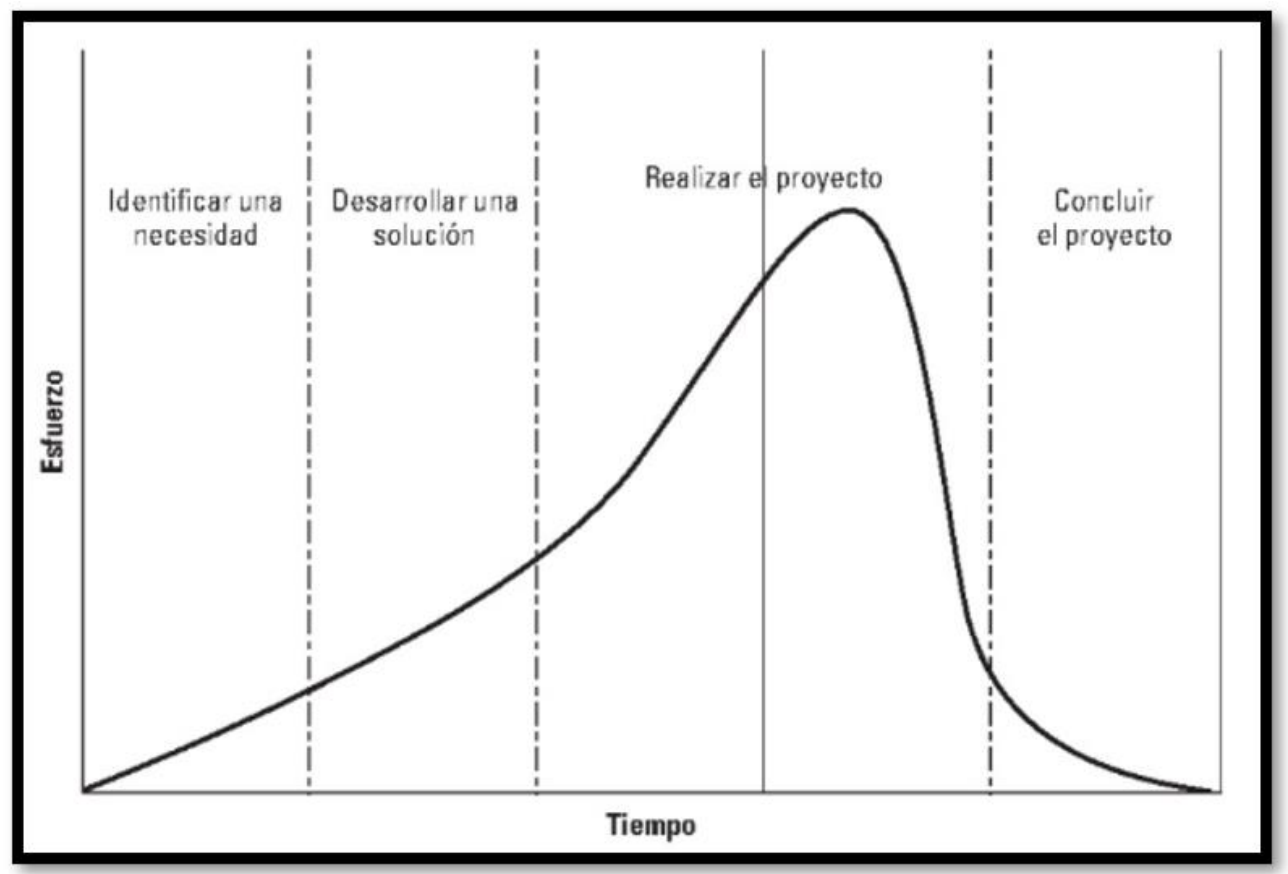

Fuente: Clements y Gido (2012, p. 9)

\section{Autoconstrucción de vivienda asistida}

Las carencias económicas de las familias de Acamixtla son el reflejo de que no hay un crecimiento y desarrollo para estas personas, situación que se vuelve más difícil, ya que, por cuestiones geográficas, entre otras, no cuentan con los servicios necesarios para salir adelante como localidad, por ejemplo: agua potable, drenaje, energía eléctrica, carreteras en buen estado; no hay hospitales, escuelas, puentes de comunicación terrestre, entre otros. En resumen, se trata de dos pilares que se encuentran totalmente 


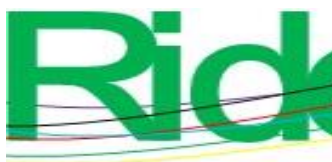

Revista Iberoamericana para la

Investigación y el Desarrollo Educativo ISSN 2007 - 7467

desconfigurados: 1) la gente que no quiere migrar de ahí aun sabiendo que es una vía para tener una mejor vida y 2) las condiciones naturales del poblado que impiden el desarrollo social.

Ahora bien, antes de continuar con la metodología de este trabajo vale la pena citar al pie de la letra las definiciones que otorga la Ley de Vivienda (14 de mayo de 2019) de conceptos que hemos venido tocando:

I. Autoproducción de vivienda: el proceso de gestión de suelo, construcción y distribución de vivienda bajo el control directo de sus usuarios de forma individual o colectiva, la cual puede desarrollarse mediante la contratación de terceros o por medio de procesos de autoconstrucción;

II. Autoconstrucción de vivienda: el proceso de construcción o edificación de la vivienda realizada directamente por sus propios usuarios, en forma individual, familiar o colectiva;

III. Estímulos: las medidas de carácter jurídico, administrativo, fiscal o financiero que establezcan los diferentes órdenes de gobierno para promover y facilitar la participación de los sectores social y privado, en la ejecución de acciones, procesos o programas habitacionales;

IV. Espacios habitables: el lugar de la vivienda donde se desarrollan actividades de reunión o descanso, que cuenten con las dimensiones mínimas de superficie, altura, ventilación e iluminación natural, además de contar como mínimo con un baño, cocina, estanciacomedor y dos recamaras, de conformidad con las características y condiciones mínimas necesarias que establezcan las leyes y las normas oficiales mexicanas (p. 2).

\section{Metodología}

El presente trabajo se realizó bajo un enfoque cualitativo, con un alcance descriptivo. Para esta investigación, se tomó en cuenta el método bibliográfico, histórico, analítico y descriptivo con el objetivo de aplicar esta información en áreas específicas, de acuerdo con las características etnográficas, narrativas y fenomenológicas.

A partir de un diseño etnográfico, se describe e interpreta la historia de la localidad de Acamixtla, una población marginal, de pobreza extrema, y los subsistemas identificados como variables de transformación social. "Los participantes en acción son 
las circunstancias comunes o especiales que implica un proceso cultural porque se estudian temas y patrones referidos a las culturas" (Hernández, Fernández y Baptista, 2014, p. 482).

El diseño narrativo "entiende la sucesión de los hechos y situaciones a través de las vivencias contadas, entendidas como historias narradas por los participantes" (Hernández et al., 2014, p. 487).

La experiencia compartida de las familias en pobreza extrema tiene en el diseño fenomenológico el propósito de "comprender las experiencias de las personas con respecto al fenómeno y descubrir denominadores comunes entre ellos" (Hernández et al., 2014, p. 493).

\section{Resultados}

A continuación, se presentan los resultados de la investigación: todos los ciudadanos tienen derecho a una mejor calidad de vida y esto se va a lograr iniciando por la interacción social con los de su misma localidad, es decir, dar apertura a un abanico de opciones sociales como la participación social, la participación política, la participación cultural y la participación laboral. El resultado de dichas participaciones se verá reflejado en su poder decisorio.

El Inegi (2020) informa que Taxco presenta una población total de 105 586, de los cuales 5301 viven en Acamixtla, lo cual corresponde a la muestra por grupo de interés.

Tabla 1. Detalle del cálculo de la muestra por sujetos de estudio.

\begin{tabular}{|c|c|c|c|}
\hline Sujeto de estudio & Población & Porcentaje & Resultado \\
\hline Habitantes de Acamixtla & 5301 & $1.5 \%$ & 80 \\
\hline
\end{tabular}

Fuente: Elaboración propia con base en datos del Inegi (2020)

Una vez analizada la información anterior, se interpreta que de la muestra 80 personas son las que viven en pobreza extrema. Cada familia está compuesta por entre 8 y hasta 11 miembros. 
Tabla 2. Familias en pobreza extrema

\begin{tabular}{|c|c|c|c|}
\hline Sujeto de estudio & Población & Familias & $\begin{array}{c}\text { Promedio de miembros } \\
\text { por familia }\end{array}$ \\
\hline $\begin{array}{c}\text { Habitantes de Acamixtla } \\
\text { en pobreza extrema }\end{array}$ & 80 & 8 & De 8 a 11 \\
\hline
\end{tabular}

Fuente: Elaboración propia con base en datos del Inegi (2020)

A partir de la tabla anterior se deduce que cada familia está compuesta por arriba de siete miembros, y si a ello se le suma que no cuentan con los ingresos económicos suficientes para tener una vivienda con los servicios básicos, da como resultado condiciones de vida no dignas, incluyendo una vivienda no apta a sus necesidades.

Cabe mencionar que la pobreza moderada se presenta cuando hace falta una variable de las mostradas en la tabla 3 y la pobreza extrema es cuando se presenta más de una. Para esta investigación se utilizaron 6 variables.

Tabla 3. Listado de variables de transformación social

\begin{tabular}{|c|c|}
\hline Núm. & Variable \\
\hline 1 & Rezago educativo \\
\hline 2 & Acceso a los servicios de salud \\
\hline 3 & Acceso a la seguridad social \\
\hline 4 & Acceso a la alimentación \\
\hline 5 & Calidad de espacios de la vivienda \\
\hline 6 & Acceso a los servicios básicos de la \\
& vivienda \\
\hline
\end{tabular}

Fuente: Elaboración propia con base en datos del Inegi (2020)

Este poblado engrosa las filas de la pobreza extrema al presentar más de dos variables, todo lo cual se refleja en tener una vivienda insegura, construida con materiales de mala calidad, con espacio insuficiente y sin contar con la disponibilidad de servicios básicos. Por tener un alto grado de marginación, los materiales naturales como la piedra caliza, la palma, el ladrillo, el adobe, el teyolote, no representan un desembolso, porque son insumos que la madre naturaleza proporciona, pero también hay materiales que compran como la lámina de cartón, tejas, o cualquier otro, pero de muy baja o de mala calidad, que utilizan para la edificación, ampliación o remodelación de su vivienda, con el objetivo de reducir el déficit de vivienda y el hacinamiento. 


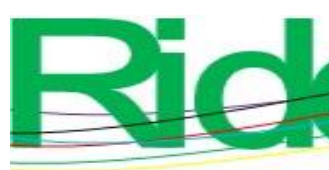

Revista Iberoamericana para la Investigación y el Desarrollo Educativo ISSN 2007 - 7467

Para apoyar a esta población marginal, se difunde el término de autoproducción asistida, que fomenta nuevos quehaceres en las formas de construcción y sentir la satisfacción de que está su espacio para vivir o para mejorar un poco su hábitat. Este apoyo tiene una estructura secuencial de actividades aplicadas en la administración de proyectos reflejadas en el ciclo de vida del proyecto, tal y como se presenta en la tabla 4.

Tabla 4. Etapas del ciclo de la vida del proyecto, desarrollo y resultado

\begin{tabular}{|c|l|l|}
\hline $\begin{array}{c}\text { Etapa del ciclo de } \\
\text { proyecto }\end{array}$ & \multicolumn{1}{|c|}{ Desarrollo } & \multicolumn{1}{|c|}{ Resultado } \\
\hline Inicio del proyecto & $\begin{array}{l}\text { Diagnóstico actual de la } \\
\text { vivienda }\end{array}$ & $\begin{array}{l}\text { Análisis de un asesor } \\
\text { técnico para determinar el } \\
\text { presupuesto }\end{array}$ \\
\hline Organización preparación & Planificación de las tareas & Supervisión de la obra \\
\hline Ejecución del trabajo & $\begin{array}{l}\text { Ejecución del trabajo } \\
\text { relacionado con las tareas }\end{array}$ & Entregable de recursos \\
\hline Cierre del proyecto & $\begin{array}{l}\text { Liberación de los recursos } \\
\text { para la construcción }\end{array}$ & $\begin{array}{l}\text { Vivienda sólida y con } \\
\text { recursos básicos existentes }\end{array}$ \\
\hline
\end{tabular}

Fuente: Elaboración propia con base en PMI (2017)

Para arribar progresivamente al logro del derecho de la vivienda adecuada se consideran tres estrategias: 1) atender a la población con mayor rezago, 2) contribuir en la solución de grandes desafíos nacionales en materia urbana y de bienestar social y 3) fortalecer e incrementar intervenciones que favorezcan la producción social de vivienda asistida (Sedatu, 26 de junio de 2020). 
Figura 6. Elementos de una vivienda

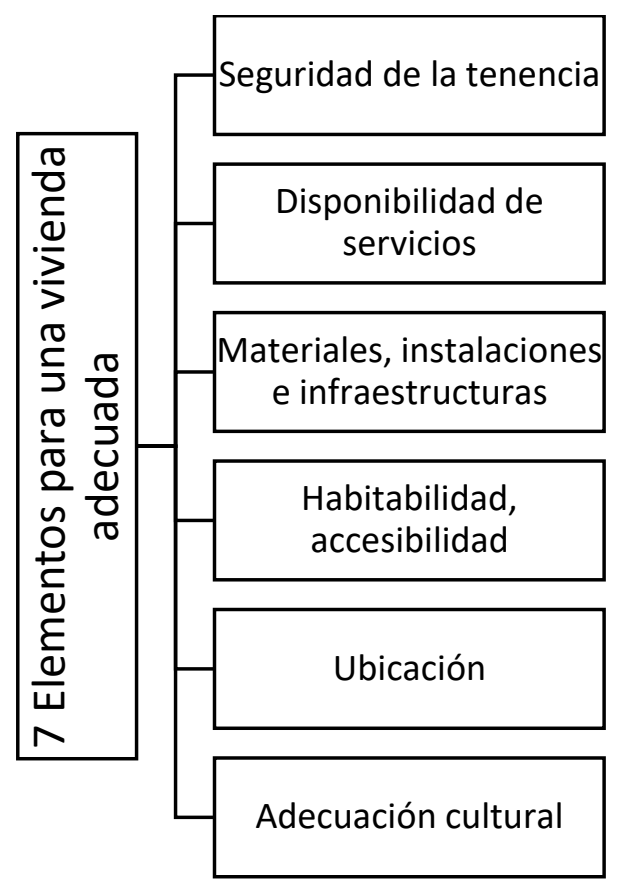

Fuente: Elaboración propia con base en Sedatu (2019)

Todos los integrantes de cada familia son partícipes como autoproductores y también como autoconstructores, porque su casa la van levantando de acuerdo con los espacios que quieran ampliar o utilizar, la satisfacción que tienen por saber que todo está trabajado con sus propias manos, pero guiados con la asesoría de un experto.

Tabla 5. Ventajas en la autoconstrucción asistida

\begin{tabular}{|l|l|}
\hline \multicolumn{1}{|c|}{ Ventajas } & \multicolumn{1}{c|}{ Ejecución } \\
\hline Menos gastos & Las familias utilizan su propio terreno para construir. \\
\hline Participación social & $\begin{array}{l}\text { Los integrantes de una familia y demás miembros } \\
\text { comunitarios son partícipes de su producción y de la } \\
\text { autoconstrucción. }\end{array}$ \\
\hline Diseño propio & $\begin{array}{l}\text { Se amolda a las costumbres, gustos y necesidades de } \\
\text { la localidad. }\end{array}$ \\
\hline Materiales de calidad & $\begin{array}{l}\text { Insumos que resistan los daños de los fenómenos } \\
\text { naturales. }\end{array}$ \\
\hline Servicios básicos & $\begin{array}{l}\text { Agua potable, energía eléctrica, drenaje, recursos } \\
\text { indispensables en el día a día. }\end{array}$ \\
\hline Asesoría técnica & Supervisa la estructura de la vivienda. \\
\hline
\end{tabular}

Fuente: Elaboración propia con base en PMI (2017) 
Una casa de autoproducción asistida abre el camino para fortalecer el desarrollo social en materia de vivienda y cumplir con los requerimientos mínimos de habitabilidad, así como satisfacer las necesidades inmediatas.

\section{Discusión}

Asentarse en lugares que no cumplan con una planeación, organización, dirección y control urbano tiene como resultado la irregularidad de los límites en espacios de vivienda. En esta misma definición, un asentamiento irregular es una vivienda o grupo de viviendas que carece de uno de los siguientes cinco elementos: 1) acceso a agua potable, 2) drenaje, 3) seguridad en la tenencia, 4) durabilidad de la vivienda (materiales de desecho y también incluye la localización en zonas de riesgo) y 5) área suficiente para vivir (hacinamiento) (Turkstra y Raithelhuber, 2005, citados en Ruíz, 2015).

Guerrero es un estado de alta marginación, catalogado generalmente como un estado pobre. Y esta información se corrobora con los datos arrojados por el Coneval en el 2020. Se puede observar en la figura 1 que Guerrero, a nivel nacional, al año 2008 presentaba 773100 habitantes con carencia social y 843300 en 2018, es decir, 70200 habitantes más en relación con el 2008.

Figura 7. Número de habitantes en Guerrero con carencia social

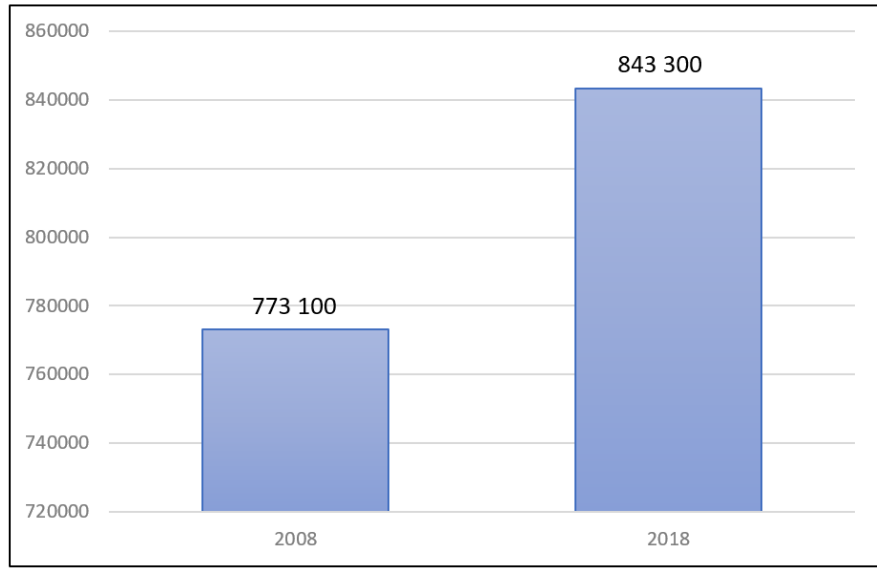

Fuente: Elaboración propia con datos de Coneval (2020)

La población en Guerrero con carencia social en 2008 era de 773100 habitantes, que representaban $22.8 \%$ del total de la población guerrerense; en 2018 hay un incremento de esta población a 70200 habitantes, que representan $23.2 \%$ (véase figura $8)$. 
Figura 8. Porcentaje de la población en Guerrero con carencia social

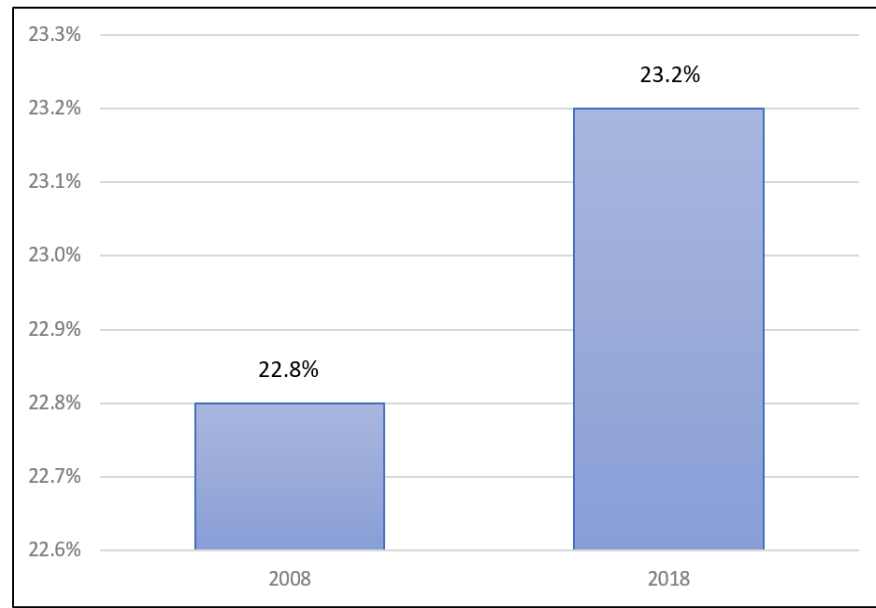

Fuente: Elaboración propia con datos de Coneval (2020)

Guerrero presenta, a nivel nacional, una población total de 3541 000; Taxco tenía un total de 105586 habitantes al año 2020. La figura 9 lo representa.

Figura 9. Población nacional de Guerrero y Taxco

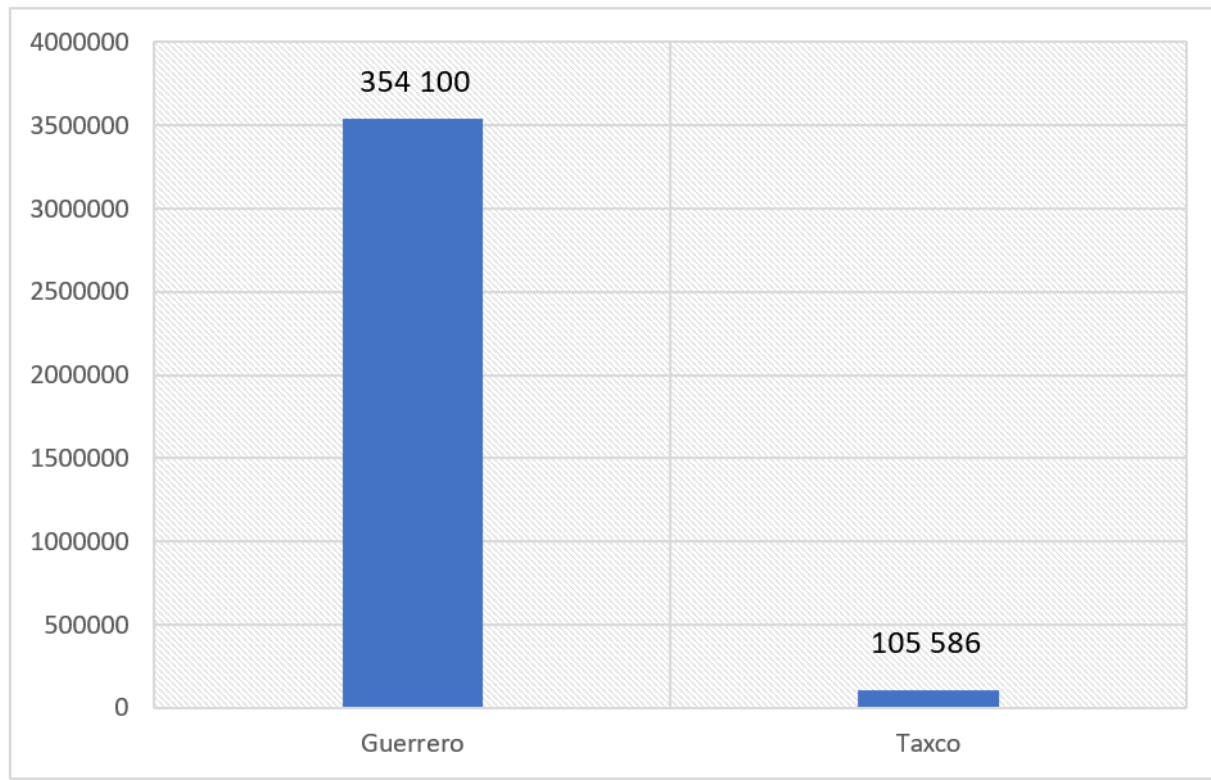

Fuente: Elaboración propia con datos de Coneval (2020)

Acamixtla al año 2020 tenía un total de 5391 habitantes, es decir, 1.5\% de los 105 586 habitantes en Taxco. La figura 10 lo representa. 
Figura 10. Número de habitantes en Taxco y el poblado de Acamixtla

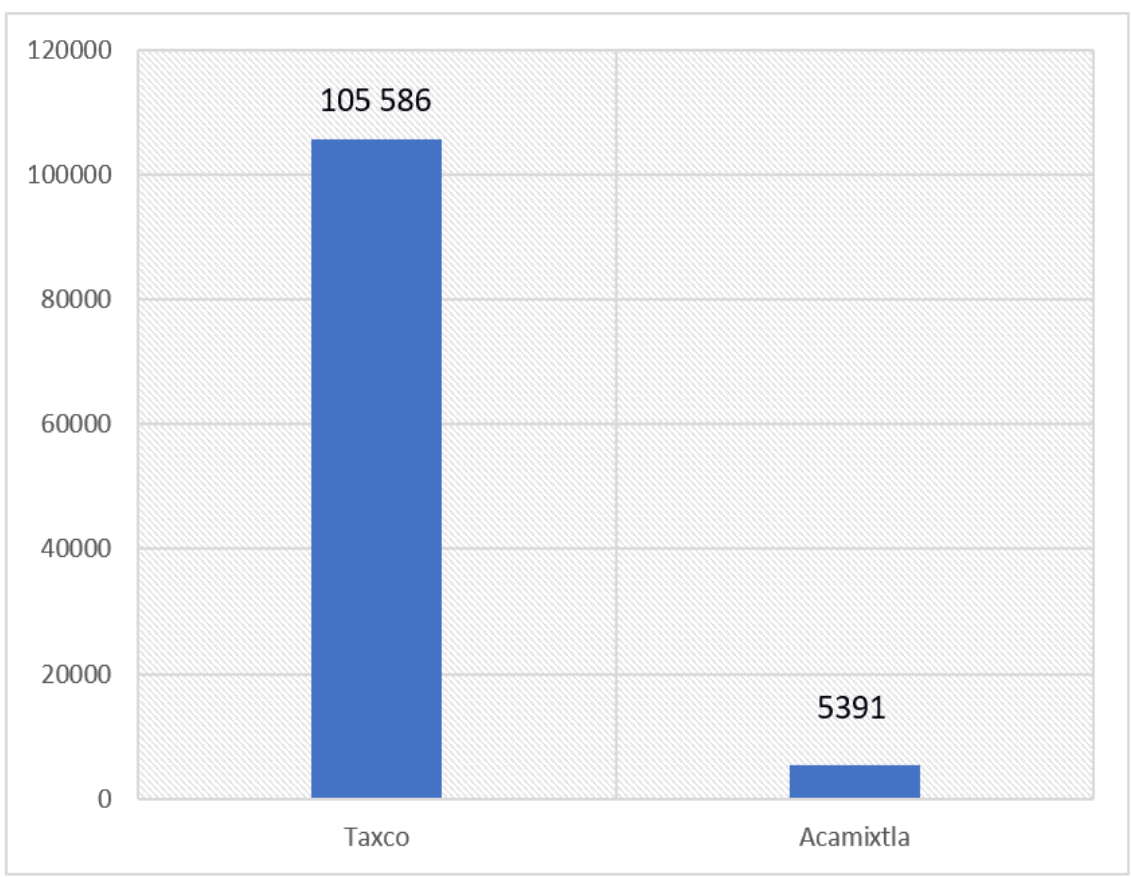

Fuente: Elaboración propia con datos de Coneval (2020)

La población de la periferia de Acamixtla, representada por 80 personas, corresponde a $1.5 \%$ de los 5301 habitantes acamixtlecos. Ocho familias viven en pobreza extrema, tienen una vivienda que ya no puede seguir siendo habitada, por no tener el espacio suficiente para el número de personas que forman cada familia. La figura 11 lo representa.

Figura 11. Población de Acamixtla

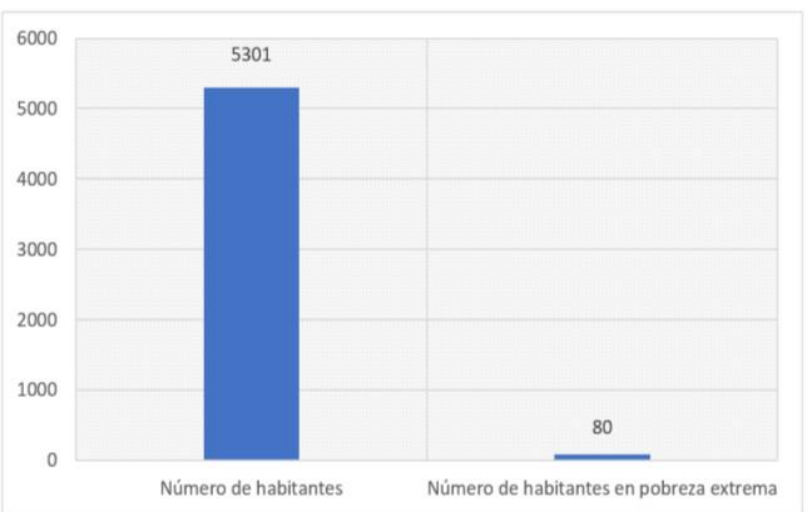

Fuente: Elaboración propia con datos de Coneval (2020)

Uno de los principales problemas en materia de vivienda en México es el rezago habitacional, el cual se refiere a la carencia de vivienda, vivienda con hacinamiento o viviendas construidas con materiales inadecuados (Salinas, 2016, citado en Mondragón, 2019). Los indicadores de carencia social en Guerrero por un periodo de 10 años no presentan mejoría en sus condiciones de vida. La figura 12 lo representa. 
Figura 12. Indicadores de carencia social en Guerrero

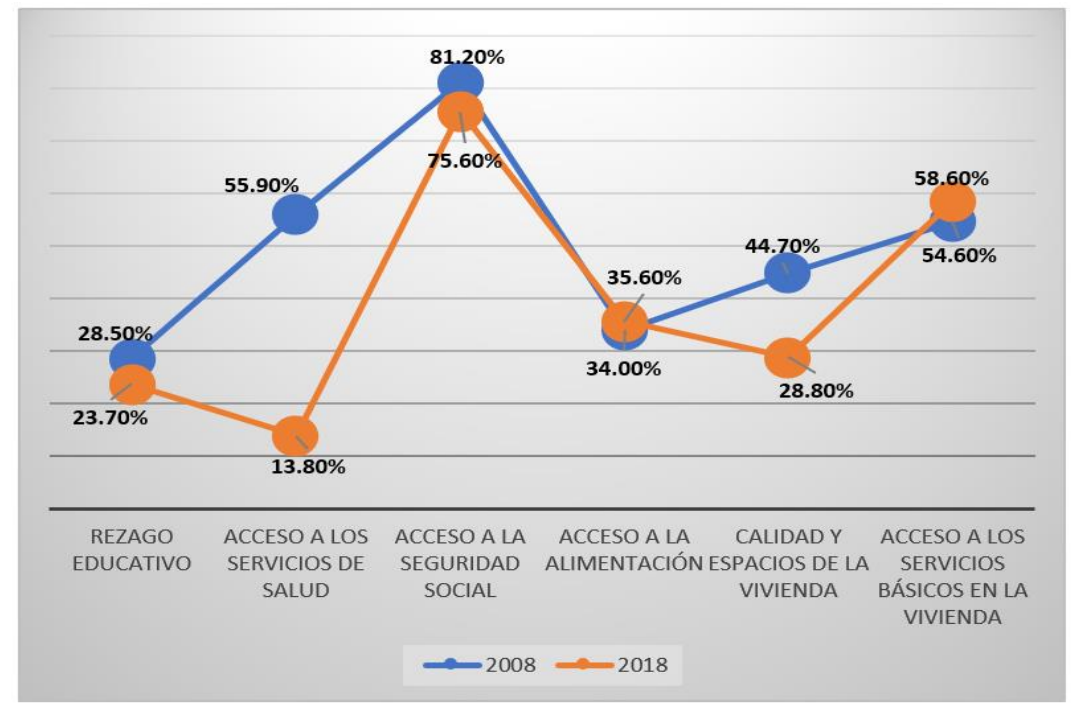

Fuente: Elaboración propia con datos de Coneval (2020)

- Indicador 1. Rezago educativo: en 2008 tiene $28.50 \%$, para el 2018 hay un ligero decremento, $23.70 \%$, esto quiere decir que algunas personas tuvieron la oportunidad de ir a la escuela.

- Indicador 2. Acceso a los servicios de salud: en 2008 era de $55.90 \%$ y en 2018 solo $13.80 \%$ ya podía disfrutar de este derecho, solicitar medicamentos o recibir atención médica.

- Indicador 3. Acceso a la seguridad social: en 2008 se registraron cifras de $81.20 \%$ y en 2018 de $75.60 \%$, lo que significa una ligera disminución, $5.60 \%$; estadísticamente, en un margen de 10 años es igual a $0.56 \%$ de mejora por año para recibir protección social y económica por perder el empleo.

- Indicador 4. Acceso a la alimentación: con un resultado en 2008 de $35.60 \%$ y al 2018 de $35.60 \%$.

- Indicador 5. Calidad y espacios de la vivienda: $44.70 \%$ en 2008 y una disminución de $22.80 \%$ al 2018, es decir, aunque aparentemente hay un descenso que favorezca la calidad en la vivienda y su distribución, para las familias de pobreza extrema en Acamixtla no existe mejora en este indicador.

- Indicador 6. Acceso a los servicios básicos en la vivienda: la electricidad, drenaje y agua potable en 2008 es de $54.60 \%$ y en 2018 de $58.60 \%$, lo que afecta más a la población.

El resultado de la medición de los seis indicadores al 2020 muestra, una vez más, que Guerrero se encuentra en el lugar tres a nivel nacional, con un grado de rezago social muy alto. Estos datos se ven reflejados en la figura 13. 


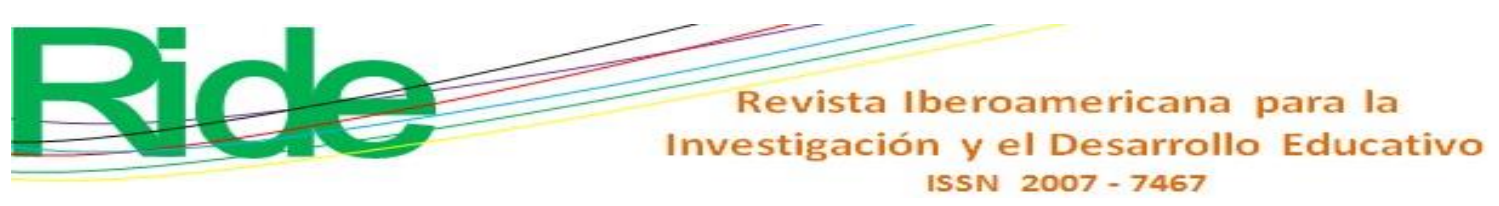

Figura 13. Rezago social de la población nacional total

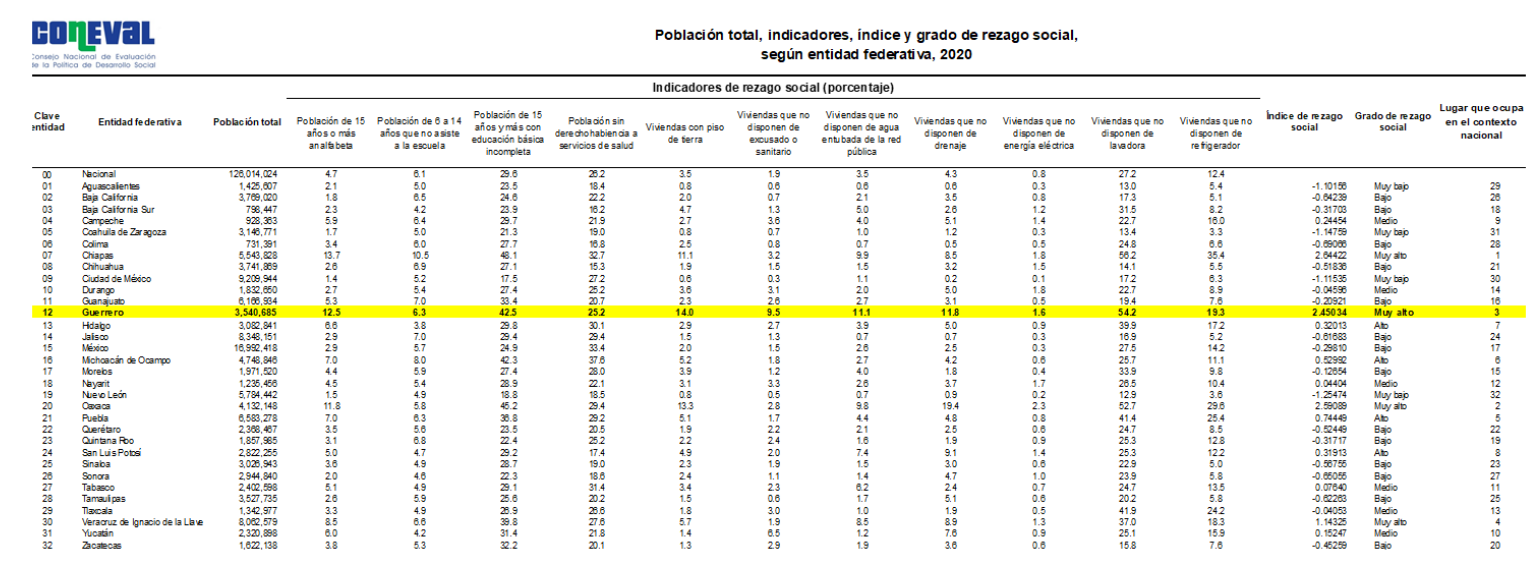

Fuente: Coneval (2020)

Las limitaciones presentadas en el objeto de estudio son las costumbres arraigadas y la desconfianza en los programas gubernamentales. Los habitantes que pueden tener el beneficio de la vivienda asistida solicitan que el apoyo no esté sujeto en hacerles cambiar sus costumbres: el director de la administración de proyecto debe comunicar al técnico o asesor del proyecto de vivienda informar a los beneficiarios las ventajas que ofrece este programa.

\section{Conclusiones}

Los indicadores de carencia social que se presentan en Acamixtla están reflejados de forma negativa en las ocho familias asentadas en lo alto de esta localidad. En relación con la situación de pobreza presente en el poblado marginal de Acamixtla, los datos fueron recabados a través de testimonios de los propios habitantes para dar a conocer que su vivienda ya no es apta para seguir siendo habitada, tanto por la reducción de espacios como por el riesgo alto que tenga la vivienda. De acuerdo con los indicadores de carencia social, es decepcionante que ninguna de las ocho familias tenga un indicador de carencia social en estatus de cumplimiento. La falta de todos los indicadores profundiza el abismo social, e impide tener una vivienda digna de habitar.

El Gobierno presenta disposición para participar en un proyecto de reconstrucción, remodelación o la construcción de una vivienda nueva. Esta actividad la supervisó el especialista en materia de construcción. La administración del proyecto se desarrolló en colaboración con todos los miembros de cada familia, quienes trabajan muy motivados para romper estos patrones de una mala calidad de vida. Es fundamental y prioritario construir una vivienda que favorezca un nuevo cambio de vida y que les dé la oportunidad de salir adelante. 
García, B. (2010). Vivienda social en México (1940-1999) actores públicos, económicos y sociales. Cuadernos de Vivienda y Urbanismo, 3(5). Recuperado de https://revistas.javeriana.edu.co/index.php/cvyu/article/view/5527.

Garrido, A. y Carrillo, J. (2013). Programación reactiva en la administración de proyectos: aproximación conceptual y aplicaciones prácticas. Revista Escuela de Administración de Negocios, (74), 73-85. Recuperado https://journal.universidadean.edu.co/index.php/Revista/article/view/737/730.

Gido, J. y Clements, J. P. (2012). Administración exitosa de proyectos (5. ${ }^{\text {a }}$ ed.). Ciudad de México, México: Cengage Learning. Recuperado de http://190.116.26.93:2171/mdv-biblioteca-virtual/libro/documento/d39rv2J4jG9ONG-Letvsu_ADMINISTRACION_EXITOSA_DE_PROYECTOS.pdf. Gutiérrez, R., Díaz, K. y Román, R. (2016). El concepto de familia en México: una revisión desde la mirada antropológica y demográfica. Ciencia Ergo Sum, 23(3), 219-230.

Hernández, R., Fernández, C. y Baptista, P. (2014). Metodología de la investigación. México: McGraw-Hill Education.

Instituto Nacional de Estadística y Geografía [Inegi]. (2016). Áreas geográficas. Guerrero (12). https://www.inegi.org.mx/app/buscador/default.html?q=guerrero\#tabMCcollapse -Indicadores.

Krajewski, L., Malhotra, M. y Ritzman, L. (2008). Administración de operaciones. Procesos y cadenas de valor (8. ${ }^{a}$ ed.). Naucalpan de Juárez, México: Prentice Hall. Recuperado de https://www.gob.mx/cms/uploads/attachment/file/566458/Administracion_De_O peraciones___LEE_J._K-comprimido.pdf.

Ley de Vivienda. (14 de mayo de 2019). Diario Oficial de la Federación. Recuperado de http://www.diputados.gob.mx/LeyesBiblio/pdf/LViv_140519.pdf.

Medianero, B. D. (2016). Productividad total. Teoría y métodos de medición. Perú: Editorial Macro.

México Real. (2017). Estado de Guerrero de la República Mexicana. Recuperado de https://mr.travelbymexico.com/688-estado-de-guerrero/.

Mondragón, S. (2019). Vivienda de interés social e interés social progresiva. Caso conjunto urbano habitacional Geovillas el Nevado, Almoloya de Juárez, Estado 
de México, 2003-2019. (Tesis de licenciatura). Universidad Autónoma del Estado de México, Toluca. Recuperado http://hdl.handle.net/20.500.11799/104532.

Ortiz, J. y Ríos, H. (2013). La pobreza en México, un análisis con enfoque multidimensional. Análisis económico, 38(69), 189-218.

Organización de las Naciones Unidas [ONU]. (s. f.). Acabar con la pobreza. Recuperado de https://www.un.org/es/global-issues/ending-poverty.

Organización de las Naciones Unidas [ONU]. (2017). Nueva Agenda Urbana. Quito, Ecuador: Secretaría de Habitat III. Recuperado de http://uploads.habitat3.org/hb3/NUA-Spanish.pdf.

Project Management Institute [PMI]. (2013). Guía de los fundamentos para la gestión de proyectos. Guía del PMBOK (5. ${ }^{\mathrm{a}}$ ed.). Estados Unidos: Project Management Institute.

Project Management Institute [PMI]. (2017). Guía de los fundamentos para la gestión de proyectos. Guía del PMBOK (6. ${ }^{\mathrm{a}}$ ed.). Estados Unidos: Project Management Institute.

Quezada, G. (2012). Gestión de proyectos de cooperación: un repaso a la teoría sobre el PMBOK. Recuperado de https:/www.ucipfg.com/Repositorio/MIA/MIA10/Unidad01/003.pdf.

Ruíz, I. E. (2015). Identificación de asentamientos irregulares y diagnóstico de sus necesidades de infraestructura en Ciudad Juárez, Chihuahua, México. Investigaciones Geográficas, (87), 88-101. Recuperado de http://www.investigacionesgeograficas.unam.mx/index.php/rig/article/view/4179 3/41692.

Sánchez, J. (2009). ¿La vivienda “social” en México. Pasado - presente - ¿futuro? México: JSA.

Secretaría de Desarrollo Agrario, Territorial y Urbano [Sedatu]. (2019). Programa Nacional de Vivienda 2019-2024. Ciudad de México, México: Secretaría de Desarrollo Agrario, Territorial y Urbano. Recuperado de https://www.gob.mx/cms/uploads/attachment/file/513675/Programa_Nacional_d e_Vivienda_2019-2024.pdf.

Secretaría de Desarrollo Agrario, Territorial y Urbano [Sedatu]. (2020). Manual de autoconstrucción. Ciudad de México, México: Secretaría de Desarrollo Agrario, Territorial y Urbano. Recuperado de 
https://www.gob.mx/cms/uploads/attachment/file/561604/Manual_de_autoconstr uccio_n.pdf.

Secretaría de Desarrollo Agrario, Territorial y Urbano [Sedatu]. (26 de junio de 2020). Acuerdo por el que se modifican las Reglas de Operación del Programa de Vivienda Social para el ejercicio fiscal 2020, publicadas el 4 de febrero de 2020.

Diario Oficial de la Federación. Recuperado de https://www.gob.mx/conavi/documentos/reglas-de-operacion-del-programa-devivienda-social-2020.

Shek, I. M. (2013). El enfoque de gestión de proyectos en las organizaciones dedicadas a proyectos de investigación. Caso: Grupo de Investigaciones GIRH. Revista Escuela de Administración de Negocios, (74), 158.

VH Construcciones y Asesoría en Ingeniería. (2009). Manifestación de impacto ambiental hidráulico modalidad particular para la planta de tratamiento de aguas residuales de la localidad de Acamixtla, municipio de Taxco de Alarcón, estado de Guerrero. México: VH Construcciones y Asesoría en Ingeniería. Recuperado de http://sinat.semarnat.gob.mx/dgiraDocs/documentos/gro/estudios/2010/12GE201 0HD012.pdf. 


\begin{tabular}{|l|l|}
\hline Rol de Contribución & Autor (es) \\
\hline Conceptualización & Luis Alberto Salgado Cabrera. \\
\hline Metodología & Lucero Way Mendez. \\
\hline Software & Levid Ezer Nava Solis. \\
\hline Validación & Luis Alberto Salgado Cabrera, Levid Ezer Nava Solis. \\
\hline Análisis Formal & Levid Ezer Nava Solis. \\
\hline Investigación & Jacqueline Bringas Ramírez. Lucero Way Mendez. \\
\hline Recursos & Luis Alberto Salgado Cabrera. Levid Ezer Nava Solis. \\
\hline Curación de datos & Levid Ezer Nava Solis. Lucero Way Mendez. \\
\hline $\begin{array}{l}\text { Escritura - Preparación del } \\
\text { borrador original }\end{array}$ & $\begin{array}{l}\text { Luis Alberto Salgado Cabrera Jacqueline Bringas Ramírez. } \\
\text { Lucero Way Mendez. }\end{array}$ \\
\hline $\begin{array}{l}\text { Escritura - Revisión y } \\
\text { edición }\end{array}$ & \begin{tabular}{l} 
Levid Ezer Nava Solis. Lucero Way Mendez. \\
\hline Visualización
\end{tabular} \\
\hline Supervisión & Jacqueline Bringas Ramírez. \\
\hline Administración de Proyectos & Lucero Way Mendez. Luis Alberto Salgado Cabrera. \\
\hline Adquisición de fondos & $\begin{array}{l}\text { Luis Alberto Salgado Cabrera. Jacqueline Bringas Ramírez. } \\
\text { Levid Ezer Nava Solis. Lucero Way Mendez. }\end{array}$ \\
\hline
\end{tabular}

